\title{
Epidermal Growth Factor Receptor Activation: An Upstream Signal for Transition of Quiescent Astrocytes into Reactive Astrocytes after Neural Injury
}

\author{
Bin Liu, ${ }^{1}$ Huiyi Chen, ${ }^{1}$ Terrance G. Johns, ${ }^{2}$ and Arthur H. Neufeld ${ }^{1}$ \\ ${ }^{1}$ Department of Ophthalmology and Visual Sciences, Washington University School of Medicine, St. Louis, Missouri 63110, and ${ }^{2}$ Ludwig Institute for \\ Cancer Research, Austin Hospital, Heidelberg 3084, Australia
}

\begin{abstract}
Modulating the behaviors of reactive astrocytes is a potential therapeutic strategy for neurodegenerative diseases. We found that upregulation and activation of the epidermal growth factor receptor (EGFR) occur in astrocytes after different injuries in optic nerves in vivo. Activation of EGFR regulates genes and cellular processes representing most major markers of reactive astrocytes and genes related with glaucomatous optic neuropathy and other neural disorders. These results suggest that activation of EGFR is a common, regulatory pathway that triggers quiescent astrocytes into reactive astrocytes in response to neural injuries in the optic nerve, and perhaps other parts of the CNS. Targeting EGFR activation using an EGFR tyrosine kinase inhibitor prevents the loss of retinal ganglion cells in a model of glaucomatous optic neuropathy. Because these inhibitors are currently used clinically, our results present an approach to reactive astrocytes as a potential new target for the treatment of neurodegenerations.
\end{abstract}

Key words: astrocyte; epidermal growth factor receptor; neural injury; neurodegeneration; gene microarray; optic nerve

\section{Introduction}

In response to a variety of neural damages in the CNS, quiescent astrocytes become reactive astrocytes. Astrocytes are the major glial subtype and are important effectors that participate in the pathogenesis of numerous neural disorders (Miller, 2005), including trauma (Norton, 1999), stroke (Swanson et al., 2004), aging (Luo and Roth, 2000), and developmental, genetic, idiopathic or acquired neurodegenerative diseases (Abraham, 2001; Teismann et al., 2003; Barbeito et al., 2004) Modulating the behaviors of reactive astrocytes is increasingly thought to be a therapeutic strategy for the treatment of CNS disorders (Tacconi, 1998; Neufeld et al., 1999; Mrak and Griffin, 2001; Wyss-Coray and Mucke, 2002; Neufeld and Liu, 2003b).

Numerous publications use optic nerve injury as a model of neurodegeneration and regeneration. We, and others, have found that reactive astrocytes are particularly active during optic nerve degeneration and are likely to contribute to the pathogenesis of glaucomatous optic neuropathy (Hernandez, 2000; Neufeld and Liu, 2003b). Screening the regulatory pathways of reactive astrocytes (Neufeld and Liu, 2003a), we demonstrated that the epidermal growth factor receptor (EGFR) pathway is an upstream signal of astrocytes in response to elevated hydrostatic

Received March 7, 2006; revised May 25, 2006; accepted June 7, 2006.

This work was supported in part by National Institutes of Health Grant EY12017. A.H.N. is the principle investigator for the grant and the recipient of the David F. Weeks Professorship in Translational Ophthalmic Research from Research to Prevent Blindness. We thank Smita Vora and Sucharita Das for contributions in the pharmacology experiments and Stephen Turney for the confocal time-lapse imaging of living cells.

Correspondence should be addressed to Dr. Bin Liu, Department of Ophthalmology, Northwestern University School of Medicine, 303 East Superior, Tarry 13-752, Chicago, IL 60611. E-mail: b-liu@northwestern.edu.

DOI:10.1523/JNEUROSCI.1004-06.2006

Copyright $\odot 2006$ Society for Neuroscience $\quad$ 0270-6474/06/267532-09\$15.00/0 pressure and causes optic nerve astrocytes to express inducible nitric oxide synthase and cyclooxygenase-2, to change shape, to increase levels of glial fibrillary acidic protein (GFAP), to modify the extracellular matrix, and to proliferate (Liu and Neufeld, 2000, 2003, 2004a,b; Zhang and Neufeld, 2005). All of these activities are characteristic of the reactive astrocyte phenotype.

In the normal adult mammalian CNS, EGFR is absent in mature astrocytes (Gomez-Pinilla et al., 1988) but has been reported as sporadically present in astrocytes in various disorders of the CNS, ranging from tumor development to neurodegenerative diseases. EGFR (HER, c-erbB1) belongs to a family of transmembrane receptors with intrinsic tyrosine kinase activity. Rapid upregulation of EGFR and its ligands occurs in astrocytes after ischemia (Planas et al., 1998; Jin et al., 2002), axotomy (Lisovoski et al., 1997), electrolytic lesion (Junier et al., 1993), and entorhinal ablation (Nieto-Sampedro et al., 1988); in the damaged regions of brains in patients after stroke (Ferrer et al., 1996) or with Alzheimer's disease (Birecree et al., 1988); and in astrocytes of optic nerves in patients with glaucoma (Liu and Neufeld, 2003). Approximately one-third of glioblastomas have EGFR amplifications (Hayashi et al., 2004). A recent study on EGFR showed that administration of EGFR inhibitors promotes significant regeneration of injured optic nerve fibers (Koprivica et al., 2005). However, this study was limited to neurons and did not consider the possible functions of EGFR in reactive astrocytes after neural injuries.

Our study presented here shows that the EGFR pathway is an upstream signal that triggers quiescent astrocytes to become reactive astrocytes. Targeting EGFR activation prevents the loss of retinal ganglion cells in a model of glaucomatous optic neuropathy. Regulation of EGFR function in astrocytes may represent a 
new therapeutic strategy for the treatment of neurodegenerative diseases.

\section{Materials and Methods Animal models}

Wistar albino male rats, 3 months of age (200-250 g), were maintained in temperature-controlled rooms on a $12 \mathrm{~h}$ light/dark cycle. Experiments were performed in accordance with the Association for Research in Vision and Ophthalmology Statement for the Use of Animals in Ophthalmology and Visual Research. All surgical procedures were done under general anesthesia by using a mixture of $80 \mathrm{mg} / \mathrm{kg}$ ketamine (Fort Dodge Laboratories, Fort Dodge, IA) and $12 \mathrm{mg} / \mathrm{kg}$ xylazine (Butler, Columbus, $\mathrm{OH})$, given intraperitoneally.

Transient eye ischemia. Ocular ischemia was performed in 10 rats by bilateral cannulation of the anterior chambers and unilateral elevation of the intraocular pressure (IOP) above systolic blood pressure for $90 \mathrm{~min}$. The anterior chamber was cannulated with a 30 gauge needle connected to saline reservoirs placed either at the level of the eye (normal, control) or above the eye (ischemic eye). The elevated saline reservoir was held slightly above the systolic blood pressure in the rat for $90 \mathrm{~min}$ and returned to equal height as the contralateral control eye. The absence of blood flow was confirmed by ophthalmoscopic observation of the blanched fundus. Both cannulas were removed at the end of the $90 \mathrm{~min}$ ischemia period. Rats were killed at $2 \mathrm{~d}$ after ischemia.

Chronic glaucoma model. Chronic, moderately elevated IOP was produced unilaterally in 10 rats by cautery of three episcleral vessels; the contralateral eye served as the comparative control. To perform the cautery, sutures were placed in the lids to keep the eye open and in the bulbar conjunctiva to manipulate the globe. Three of the four to five major trunks formed by limbal-derived veins were exposed at the equator of the eye by incising the conjunctiva. Each vessel was lifted with a small muscle hook and cauterized by direct application of an ophthalmic, disposable cautery (model RS201; Roboz Surgical Instrument Company, Rockville, MD) against the muscle hook. Immediate retraction and absence of bleeding of the cauterized ends of the vessels were noted as successful cauterization. After surgery, the eyes were treated topically with bacitracin-neomycin-polymyxin (Pharmaderm, Melville, NY) for a few days during recovery. The IOP was measured immediately and documented every month. Rats were killed at 1 month after the surgery.

Optic nerve transection. Optic nerve transection was performed unilaterally in 10 rats. The optic nerve was exposed by longitudinal incision of the conjunctiva at $5 \mathrm{~mm}$ behind the limbus and separation of the eye retractor muscle and the perineurium. At 3-4 mm behind the globe, a small incision was cut by a sharp blade to open the optic nerve sheath, and the optic nerve was cut without damaging the central retinal blood vessels. No loss of retinal circulation was confirmed by ophthalmoscopic observation. In the control eye, the same surgery was performed, but without cutting the optic nerve. Animals were killed at $2 \mathrm{~d}$ after the surgery.

\section{Immunohistochemistry}

Eyes were enucleated and fixed in $4 \%$ paraformaldehyde, and the optic nerves were dissected free of surrounding tissues. Fixed tissues were washed in $0.2 \%$ glycine in PBS, pH 7.4, dehydrated, embedded in paraffin, and oriented for $6 \mu \mathrm{m}$ sagittal. Three sections per eye were chosen randomly for standard hematoxylin and eosin staining for observation of histological structure. The neighboring slides of each of the three sections were used for immunohistochemical studies.

The paraffin tissue sections were deparaffined, rehydrated, and washed completely with $0.1 \mathrm{M}$ glycine-Tris solution. To block nonspecific binding, the tissue sections were preincubated with $20 \%$ donkey serum at room temperature for $30 \mathrm{~min}$. Immunohistochemistry was performed using specific primary antibodies against p-EGFR, EGFR (goat polyclonal; working dilution, 1:100; Santa Cruz Biotechnology, Santa Cruz, CA), EGF (working dilution, 1:200), amphiregulin (working dilution, 1:100), or transforming growth factor- $\alpha$ (working dilution, 1:50; rabbit polyclonal; Santa Cruz Biotechnology) and using the Vectastain Elite ABC kit (Vector Laboratories, Burlingame, CA), using diaminobenzidine as a substrate. Hematoxylin was the counterstain. For immunofluorescent staining, fluorescent-conjugated secondary antibodies, goat anti-mouse Oregon green (1:400), goat anti-rabbit rhodamine red-X-conjugated IgG (1:1000), and horse anti-goat FITC-Xconjugated IgG (1:50; Invitrogen, Eugene, OR) were used. For double immunofluorescent staining, tissues were sequentially incubated with the second primary antibody and the second appropriate secondary antibody. Negative controls were performed in parallel by preincubation of the primary antibody at $4^{\circ} \mathrm{C}$ overnight with its specific blocking peptide.

\section{Immunoblot}

Rat optic nerve tissues were dissected behind the eye globe $\sim 3 \mathrm{~mm}$ long and pooled from two to three eyes. Rat optic nerve tissue was lysed in buffer (20 mM HEPES, pH 7.0, $10 \mathrm{~mm} \mathrm{KCl,} 2 \mathrm{~mm} \mathrm{MgCl}_{2}, 0.5 \%$ Nonidet P-40, $1 \mathrm{mM} \mathrm{Na}_{3} \mathrm{VO}_{4}, 1 \mathrm{~mm}$ PMSF, and $0.15 \mathrm{U} \mathrm{ml}^{-1}$ aprotinin) and homogenized. Protein concentration was determined using the Bradford colorimetric assay. Fifty micrograms of each protein lysate were loaded in each lane in sample buffer ( $2 \%$ SDS, $10 \%$ glycerol, $0.001 \%$ bromophenol blue, $1 \%$ DTT, and $0.05 \mathrm{~m}$ Tris- $\mathrm{HCl}, \mathrm{pH} 6.8$ ), separated on $10 \%$ SDSPAGE, and transferred to a nitrocellulose filter. The blots were blocked with 5\% nonfat milk in PBS and incubated with specific rabbit polyclonal antibody against EGFR (working dilution, 1:200; Santa Cruz Biotechnology), followed by peroxidase-conjugated goat anti-rabbit $\operatorname{IgG}_{2 \mathrm{a}}$ and the enhanced chemoluminescence detection system (Amersham Biosciences, Arlington Heights, IL).

\section{Primary optic nerve head astrocyte cultures}

Rat optic nerve astrocytes were derived from the anterior portions of the rat optic nerve from six Wistar rats (3 months of age; Charles River, Chicago, IL). The posterior pole of the eye was dissected, and the optic nerve head was freed from sclera and other neighboring tissues. The optic nerve head was sliced sagittally, and, under a dissecting microscope, the anterior portion of the optic nerve was carefully dissected from the preand post-laminar regions. From each eye, two or three explants of the anterior region were obtained. The explants were put into T $25 \mathrm{~cm}^{2}$ plastic tissue culture flasks, which had been conditioned with DMEM/ F-12 supplemented with $10 \%$ fetal bovine serum. The first passage cells were characterized by immunostaining of GFAP and neural cell adhesion molecule and other cellular markers (HLA-DR, $\alpha$-smooth muscle actin, and von Willebrand factor) to distinguish them from other cell types from the optic nerve. The primary cell cultures were purified for astrocytes by growing the cells for 1 week in modified astrocyte-defined, serum-free medium (ADM; Clonetics, San Diego, CA) containing forskolin, which suppresses the growth of fibroblasts. The second-passage cell cultures, which had $>95 \%$ cells positive for GFAP, were grown to $60-80 \%$ confluency and serum starved in ADM for 1 week before being used for the following experiments.

\section{Live cell imaging}

To clearly view the behavior of individual cells by confocal microscopy, primary astrocytes were transfected by plasmids expressing green fluorescent protein (GFP) protein (pmaxGFP; Amaxa, Gaithersburg, MD) using the Nucleofector kit and the Amaxa transfection machine according to the manufacturer's protocol for rat primary astrocyte cultures (Amaxa). The transfected cells were grown on poly-D-lysine-coated glass-bottom dishes (MatTek Corporation, Ashland, MA). The cell dish was put into a conditioned chamber in which the temperature and $\mathrm{CO}_{2}$ concentration were calibrated and modulated by CTI controller 3700 (Zeiss, Oberkochen, Germany) and viewed under a confocal microscope (ConfoCor 2, Axiovert 200M; Zeiss). EGF (100 ng/ml) was added to the cell media. Confocal photomicrographs were taken every $5 \mathrm{~min}$ for up to 7 h. Astrocytes not treated with EGF were similarly observed.

\section{Microarray analysis}

The concentrations of EGF as well as the exposure time used for treatment of astrocytes were chosen on the basis of our previous studies and our confocal observations of living cells. EGF (100 ng/ul) was add to the cell media, and cells were harvested for microarray analyses after 4 or $12 \mathrm{~h}$ of treatment. Having viewed the time-lapse confocal microscopy, we considered $4 \mathrm{~h}$ as a suitable time point to allow us to detect relevant changes in gene expression that were directly in response to EGF stimu- 
lation. We also performed gene profiling at $12 \mathrm{~h}$ of treatment to determine the stability and time courses of the EGF-stimulated responses.

Two independent experiments using two cell lines from two different rat donors were performed for the microarray analyses. Six samples in the control group, three samples in the $4 \mathrm{~h}$ EGF treatment group, and three samples in the $12 \mathrm{~h}$ EGF treatment group were processed for gene microarray and analyzed as follows. A total of $\sim 2 \times 10^{6}$ cells in each sample were extracted for the total RNA using RNeasy Mini kits (Qiagen, Valencia, CA) following the manufacturer's protocols. Briefly, cells were homogenized in fresh RNA lysis tissue buffer. RNA was precipitated by $70 \%$ ethanol and collected by an RNeasy spin column. After washing several times, the total RNA was eluted from the column and further purified by ethanol precipitation. The DNA-free RNA was assessed for integrity by formaldehyde/agarose gel electrophoresis and quantitated by $260 \mathrm{~nm}$ UV light absorbance.

cDNA was synthesized from $3 \mu \mathrm{g}$ of purified RNA by using the SuperScript Choice system (Invitrogen, Carlsbad, CA) and T7-(dT) ${ }_{24}$ primer. cDNA was purified by phenol/chloroform extraction and ethanol precipitation. Using the Bioarray High Yield RNA transcript labeling kit (Enzo Diagnostics, New York, NY), the biotinylated cRNA was generated by transcript labeling the purified cDNA with biotinylated UTP and CTP following the manufacturer's protocol. The labeled cRNA was purified by using the RNeasy total RNA clean-up protocol (Qiagen) and quantitated by $260 \mathrm{~nm}$ UV light absorbance. Twenty micrograms of cRNA were fragmented before hybridization. An aliquot of fragmented and unfragmented cRNA was analyzed by formaldehyde/agarose gel to ensure appropriate size distribution in the fragmented samples.

Hybridization of the labeled cRNA to rat genome chips (Rat 230_2_0; Affymetrix, Santa Clara, CA) was performed by using the GeneChip Instrument System at the GeneChip Core Facility of Washington University. The arrays was washed and stained with streptavidin phycoerythrin (Invitrogen) and scanned on a GeneArray Scanner G2500A (Agilent Technologies, Palo Alto, CA). The imaged data were captured and converted to a numerical output by using the GeneChip Analysis Suite (version 4.0) software.

\section{Microarray data statistical analysis}

The CEL files were obtained by using Affymetrix Microarray Suite software. The DNA-Chip Analyzer (Dchip, version 1.3) (Li and Hung, 2001) was used to normalize all CEL files to the baseline array and obtain the model-based expression levels using the perfect match-only model. Genes that were rated absent across all arrays were filtered out. Genes that had significant variation in expression across all arrays were selected by choosing the coefficient of variation (the ratio of the SD and the mean values of the expression of a gene across all samples) $>0.5$. The hierarchical clustering algorithm was performed on the filtered genes in control (non-EGF-treated) and EGF-treated groups to cluster genes and group samples according to the Pearson correlation coefficient between the standardized expression values of two genes or two samples. To identify differentially expressed genes, fold changes were calculated between control and EGF-treated groups and sorted for more than twofold-changed gene expressions by the lower confidence bound, which is a conservative estimate of the fold change. The identified genes were further analyzed for functional categories according to ontology gene categories using the Function expression software.

\section{Real-time reverse transcription-PCR confirmation}

One hundred nanograms of total RNA were reverse transcribed into cDNA using a one-step supertranscript kit (Bio-Rad, Hercules, CA), according to the manufacturer's instructions. The increase in fluorescence was detected in real time using the iCycler (Bio-Rad). All quantitative real-time reverse transcription (RT)-PCRs were performed in triplicate. Relative quantitation of gene expression was performed using a relative standard curve method (User Bulletin 2 of the ABI Prism 7700 Sequence Detection System; PE Applied Biosystems, San Diego, CA). A standard curve of cycle thresholds for four serial (10 times each) dilutions of the individual gene probe was established and used to calculate the relative abundance of mRNA copy number of the gene. Values were normalized to the relative amounts of $18 \mathrm{~S}$ mRNA copy number. Primers (supplemental Table S2, available at www.jneurosci.org as supplemental material) were synthesized by Integrated DNA Technologies (Coralville, IA).

\section{Pharmacology in rat glaucoma model}

There were four groups with eight rats for each group. Group 1 was not treated with any drug. Group 2 was treated with AG1478 starting on the day that the IOP was elevated by the cautery of episcleral vessels (EVC) and continued to be treated with AG1478 for 7 months. Group 3 was not treated for the first 3.5 months after elevation of the IOP and was then treated with AG1478 for 3.5 months. Group 4 was treated for 7 months with the inhibitor of inducible nitrix oxide synthase, aminoguanidine, starting on the day that the IOP was elevated by the EVC and served as a positive control.

Body weight was measured at the time of the EVC and at 3 and 7 months after the EVC. The IOP was measured at the time of the EVC and at 2, 3, 4, and 7 months after the EVC. The amount of water consumed was measured semi-weekly by weighing the water bottle before and after filling it with a set amount of water, water containing AG1478 (0.1 mM), or water containing aminoguanidine (Neufeld et al., 1999). Each animal was caged individually.

\section{Results}

\section{Upregulation of EGFR in astrocytes in vivo}

We first determined whether the appearance of EGFR in astrocytes was a common phenomenon when a neural tissue, such as the optic nerve, was damaged in different manners. We used both immunohistochemistry and immunoblot to detect the upregulation and activation of EGFR in astrocytes of the optic nerve after several different optic nerve injuries, including acute ischemia, chronic glaucoma, and optic nerve transection (Fig. 1). Labeling for EGFR was very weak or absent in normal rat optic nerves (Fig. $1 A$ ) but became significantly intense in astrocytes in optic nerves within $2 \mathrm{~d}$ after ischemia (Fig. $1 B$ ) or optic nerve transection (Fig. $1 C$ ), and within 1 month in the rat chronic glaucoma model (Fig. $1 D)$. Colocalization of EGFR and GFP (GFAP), a marker often used for demonstrating reactive astrocytes, showed that EGFR is present, presumably upregulated, in astrocytes after optic nerve ischemia, transection (data not shown), and glaucoma (Fig. 1E). We never observed EGFR associated with axons, as implicated previously (Koprivica et al., 2005). The EGFR ligands, EGF (Fig. $1 F)$, amphiregulin, and transforming growth factor- $\alpha$, were present in the normal optic nerve and after injuries (data not shown). The activation of EGFR [detected as the phosphorylated form of EGFR ( $\mathrm{p}$-EGFR) by an antibody raised against the amino acid sequence containing the phosphorylated tyrosine- 1173 residue] was absent in normal but present in astrocytes in optic nerves after ischemia, transection, and in the chronic glaucoma model (Fig. 1G). Administration of a specific inhibitor of EGFR tyrosine kinase, AG1478, significantly blocked the activation of EGFR, as shown by the absence of labeling for p-EGFR in astrocytes in the optic nerves of rats in the glaucoma model treated with AG1478 daily in drinking water (Fig. $1 \mathrm{H}$ ). By immunoblot, EGFR protein was detected in a low amount in normal optic nerve heads but was significantly increased in optic nerve heads by $2 \mathrm{~d}$ after ischemia, by $2 \mathrm{~d}$ after nerve transection, and at 1 month in the chronic glaucoma model (Fig. 1I). These results suggested that the upregulation and activation of EGFR is a common phenomenon in astrocytes in response to injuries in the optic nerve.

\section{In vitro effects of activation of EGFR on astrocyte morphology}

We next determined the behavioral characteristics of astrocytes in cultures treated with the EGFR ligand, EGF. Primary cultures of astrocytes from rat optic nerve heads contain EGFR; EGFR is 


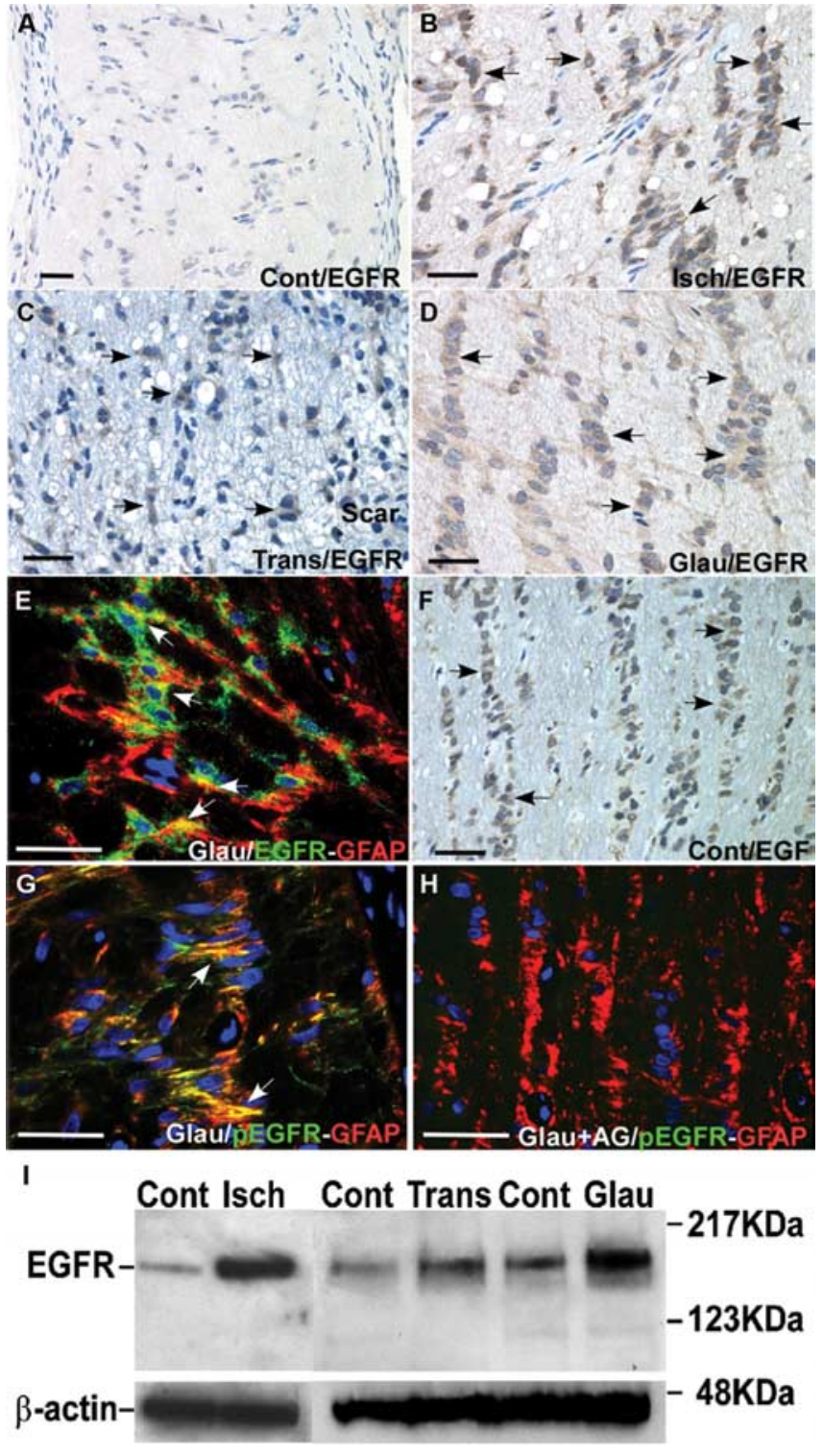

Figure 1. EGFR is expressed and activated in reactive astrocytes of optic nerves after different neural injuries. $\boldsymbol{A}-\boldsymbol{D}$, By immunohistochemistry, positive labeling of EGFR is absent or weakly present in normal optic nerves $(\boldsymbol{A})$ but significantly increased in intensity in astrocytes (arrows) in the optic nerves after ischemia $(\boldsymbol{B})$, nerve transection $(\boldsymbol{C})$, and glaucoma $(\boldsymbol{D}) . \boldsymbol{E}$, Double-immunofluorescent labeling for EGFR and GFP (GFAP) shows colocalization of EGFR (green) and GFAP (red), indicating astrocytes express EGFR. $\boldsymbol{F}$, EGFR ligand, EGF, is present in the normal optic nerve. $\boldsymbol{G}, \boldsymbol{H}$, Labeling for activated EGFR, p-EGFR, is positive and colocalized with GFAP labeling in astrocytes in optic nerves of eyes with experimentally induced glaucoma $(\boldsymbol{G})$ but appears negative in optic nerves of glaucomatous rats treated with AG1478 $(\boldsymbol{H}) . \boldsymbol{I}, \mathrm{By}$ immunoblot, EGFR protein expression increases significantly, as shown by the intense immunoblot bands, in optic nerves after eye ischemia, optic nerve transection, and glaucoma. Scale bars, $30 \mu \mathrm{m}$. Cont, Control; Isch, ischemia; Trans, transection; Glau, glaucoma.

apparently upregulated by the culture conditions (Liu and Neufeld, 2004a,b) and activated by the treatment with EGF (Liu and Neufeld, 2003). We viewed the morphological appearance and motility of these astrocytes in response to activation of EGFR by time-lapse confocal microscopy. The rat optic nerve head astrocytes that we used in culture had been sparsely transfected with an expression plasmid of GFP, so that imaging of an individual cell could be followed in a confluent cell culture. In control cultures, the cells were mostly flat, polygonal, with few short processes, and grown in monolayer. These untreated astrocytes, observed by time-lapse confocal microscopy, remained in a monolayer pattern and did not significantly change their shape or position over the $7 \mathrm{~h}$ incubation period (data not shown). In the presence of EGF (Fig. 2 and supplemental video S1, available at www.jneurosci.org as supplemental material), within $2 \mathrm{~h}$, the cells started to change shape, and after $4 \mathrm{~h}$, the cells throughout the culture were significantly elongated, had extended long processes, became highly mobile, and reorganized into a multi-layer, network-like structure. These activities continued for the next $3 \mathrm{~h}$. Obviously, activation of EGFR triggers astrocytes into a visibly active phenotype with significantly increased cell motility that is characteristic of the reactive astrocyte.

\section{Microarray analyses of astrocytes activated by EGF}

To obtain an overview of the cellular processes that are changed in astrocytes in response to activation of EGFR, we performed gene microarray analyses after treatment with EGF for 4 or $12 \mathrm{~h}$. The full CEL file data set is accessible as supplemental material in supplemental Table S1 (available at http://www.ncbi.nlm.nih. gov/projects/geo/). Quantitative real-time RT-PCR was used to confirm the reliability of the gene microarray data. Seventeen randomly selected genes matched with the data obtained from gene microarray analyses (supplemental Table S2 and Fig. S1, available at www.jneurosci.org as supplemental material), indicating the high reliability of our gene microarray data.

The microarray data showed reproducible and distinctive transcriptional profiles comparing non-EGF-treated and EGFtreated astrocytes. Replicates had correlation coefficients $>0.95$, indicating a high degree of reproducibility. To identify the distinctive transcriptional profiles, we performed unsupervised hierarchical clustering (UHC) to group the samples and cluster the genes by Dchip software (Li and Wong, 2001). This unbiased approach separated the experimental samples into two distinctive groups: the untreated (control) group and the EGF-treated group. The EGF-treated group was further divided into EGFtreated for $4 \mathrm{~h}$ and EGF-treated for $12 \mathrm{~h}$ subgroups. A UHC map is shown in Figure 3. The distinctiveness of the transcriptional profile and the time-lapse confocal microscopy of EGF-treated astrocytes compared with control astrocytes suggest that activation of EGFR switches the quiescent, cultured astrocytes into a quite different phenotype.

The transcriptional profiles were compared among control and EGF-treated astrocytes using Dchip analysis. An "EGFR pathway-regulated gene" was defined as one that differed significantly (above twofold) in expression level when comparing control and EGF-treated groups. Nine hundred eighty-five genes were identified as EGFR pathway-regulated genes at $4 \mathrm{~h}$ of EGF treatment. By extending our analyses to include gene expression at $12 \mathrm{~h}$, we found that the expression of EGFR pathway-regulated genes had different temporal patterns (supplemental Fig. S2 and Table S3, available at www.jneurosci.org as supplemental material).

Functional classification of EGFR pathway-regulated genes yielded distinct categories (supplemental Table S4, available at www.jneurosci.org as supplemental material) related to cellular processes that are characteristic of reactive astrocytes. EGFR pathway-regulated genes may support reactive astrocyte functions such as the following: (1) migration (e.g., fibroblast growth factor receptor 3; protein tyrosine phosphatase non-receptor type 1; Unc-5 homolog C; myosin; paxillin; cadherin; microtubule-associated protein-1b and -6 ; nestin; and integrin $\alpha 6$ ); (2) reorganization of the extracellular matrix (e.g., matrix metallopeptidase-3, -16, and -24; tissue inhibitor of metallopro- 
teinase; collagens XI, XV, and XVIII; laminin; chondroitin sulfate proteoglycan 2; plasminogen activator, urokinase; and hyaluronan synthase 2); and (3) cell-cell interactions (e.g., chemokine ligand- 2 and -20 ; chemotactic protein-3; monocyte to macrophage differentiation-associated; transforming growth factor $\beta 1$; endothelin receptor A; prostaglandin-endoperoxide synthase 2; phospholipase $C$; and phospholipase A), and synthesis and release of growth factors (e.g., transforming growth factor $\beta 1$; brain-derived neurotrophic factor; platelet-derived growth factor $\alpha$; fibroblast growth factor receptor-2; amphiregulin; nerve growth factor $\beta$ ).

There is also a prominently represented category of EGFR pathway-regulated genes linked to neurogenesis (e.g., brainderived neurotrophic factor; rapostlin; neuritin; nestin), suggesting possible effects on axogenesis by reactive astrocytes.

In addition, several EGFR-upregulated genes encode EGFR ligands, such as epiregulin, amphiregulin, and HB-EGF. Expression of these EGFR ligands by astrocytes after injury might establish a positive-feedback loop in an autocrine manner in reactive astrocytes to produce a chronic state of activity.

\section{Comparison of microarray data with documented markers of reactive astrocytes}

To further test the hypothesis that activation of EGFR triggers cellular pathways characteristic of reactive astrocytes, we compared the transcriptional profile of EGF-treated astrocytes to putative markers of reactive astrocytes. The documented markers of reactive astrocytes, either in vivo or in vitro, were mostly in categories of cytoskeletal filaments, growth factors/cytokines, recognition/adhesion molecules, and enzymes. Comparing these marker proteins with the gene expression profile of astrocytes treated with EGF showed that the activation of EGFR regulates the genes of many of these proteins (Table 1). Some genes characteristic of reactive astrocytes were already highly transcribed in control astrocytes in vitro before EGF treatment and were not further regulated by the EGFR pathway. Baseline expression of such genes in vitro may be attributable to astrocytes becoming partially active in response to stimuli in the cell culture environment (Eddleston and Mucke, 1993). A small number of genes characteristic of reactive astrocytes were rated absent or not included on our chips.

As indicated in Table 1, gene products, synthesized from EGFR pathway-regulated genes by reactive astrocytes, may have neurotoxic effects or block neurogenesis in certain neurological disorders. Some EGFR pathway-regulated genes, although not
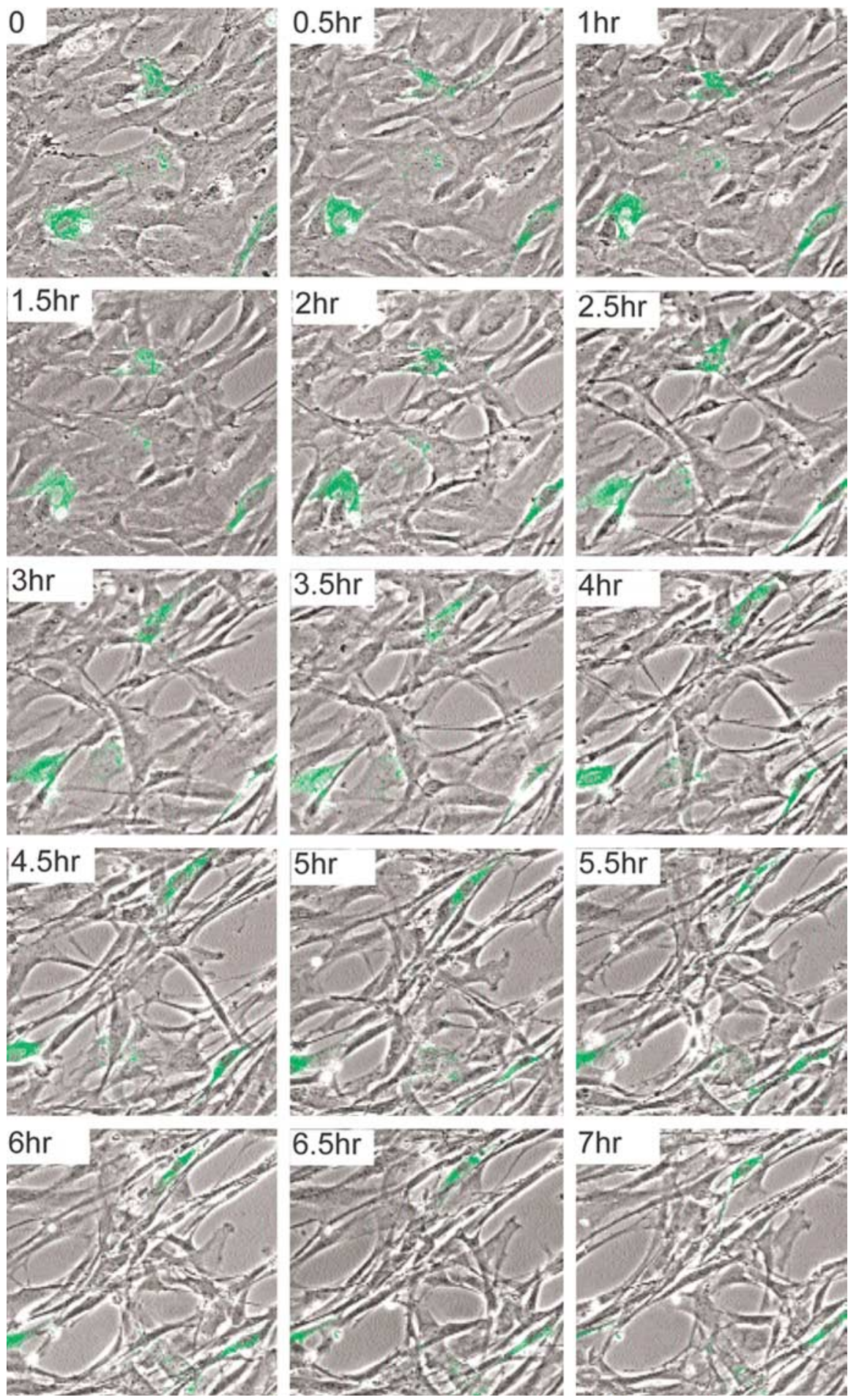

Figure 2. In vitro effects of activation of EGFR on astrocyte morphology and motility. In the presence of EGF, astrocytes rapidly became significantly elongated, extended long processes, were highly mobile, and reorganized into a multi-layer, network-like structure. Note one GFP-containing cell almost moved out of the imaging area after $7 \mathrm{~h}$ of treatment with EGF. See also supplemental video S1 (available at www.jneurosci.org as supplemental material). previously localized to reactive astrocytes, were previously reported to be associated with neural disorders. For example, oxidized low-density lipoprotein receptor 1 is associated with the late onset of Alzheimer's disease. Upregulation of serum/glucocorticoid regulated kinase coincides with the onset of dopaminergic cell death in both the 8 weeks acute and 1 year subacute Parkinson's disease models. Progressive ankylosis protein, which 


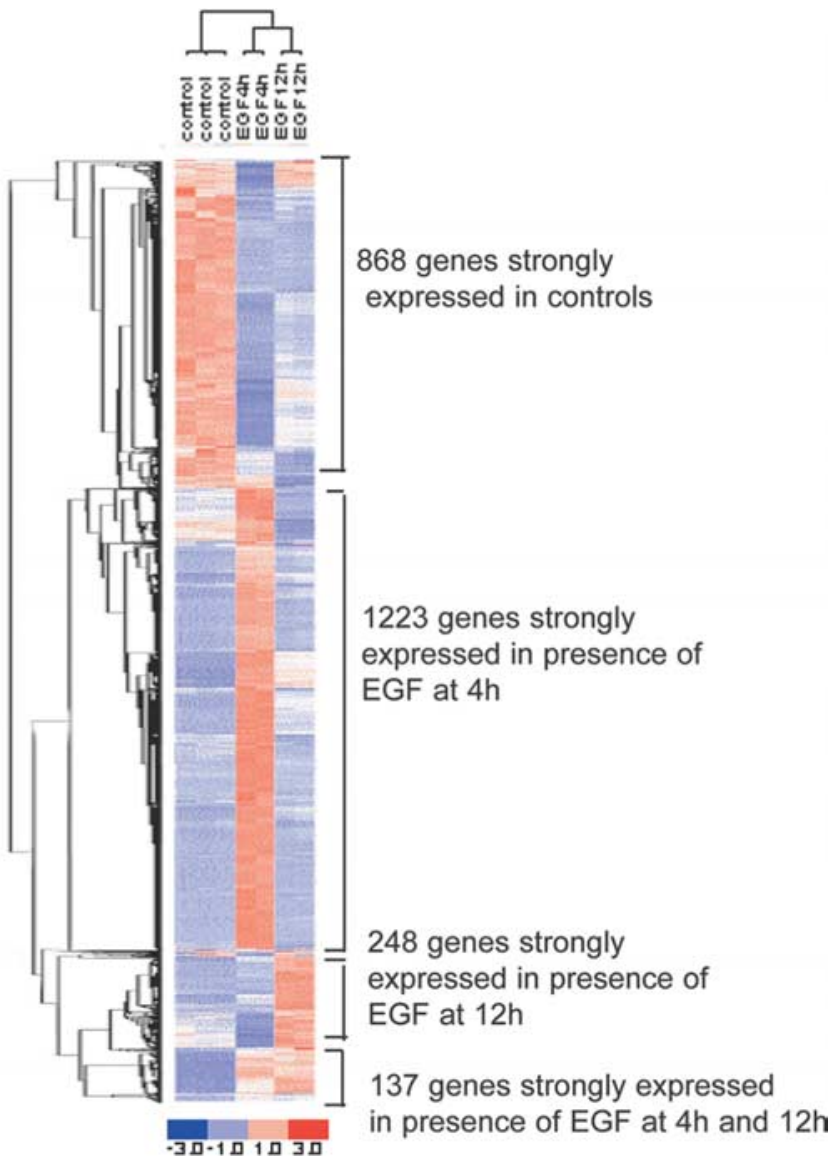

Figure 3. EGF-treated astrocytes rapidly develop distinctive transcriptional profiles compared with astrocytes not exposed to EGF. Using Dchip software, UHC was performed on the filtered genes from transcriptional profiles of controls and astrocytes treated with EGF for 4 and $12 \mathrm{~h}$. Unbiased UHC approach group controls and EGF-treated astrocytes were placed into separate groups and further divided into EGF-treated subgroups at 4 and $12 \mathrm{~h}$ of treatment. The color in the map indicates the relative expression level of each gene in each sample. Color scale: red, the expression level above the mean expression of a gene across all samples; white, the mean expression; blue, the expression lower than the mean.

regulates extracellular inorganic pyrophosphate, is expressed in the CNS and increases expression after limbic seizures.

Upregulation and activation of EGFR occur in reactive astrocytes in glaucomatous optic neuropathy (Liu and Neufeld, 2003). Three genes, including myocilin (Stone et al., 1997), optineurin (Rezaie et al., 2002), and WDR36 (Monemi et al., 2005), are identified as primary open-angle glaucoma genes. Our data show that the genes for WDR36 and an olfactomedin, which may be part of myocilin, are under the regulation of the EGFR pathway. Also, the EGFR pathway-regulated genes, such as tenascin, tissue inhibitor of metalloproteinase 1 , and matrix metalloproteinase 3 , are expressed in reactive astrocytes in glaucomatous optic neuropathy in vivo.

\section{Prevention of the loss of retinal ganglion cells by EGFR tyrosine kinase inhibitor}

From our in vitro studies, we cannot predict whether activation of EGFR stimulates destructive or protective mechanisms in vivo. However, we believe that the wide range of cellular activity stimulated by activation of EGFR in astrocytes is mostly inappropriate and unlikely to be beneficial for the surviving neurons in the injured tissue. Thus, we would predict that an inhibitor of EGFR
Table 1. List of reactive astrocytes and neural disorder-related genes regulated by the EGFR pathway

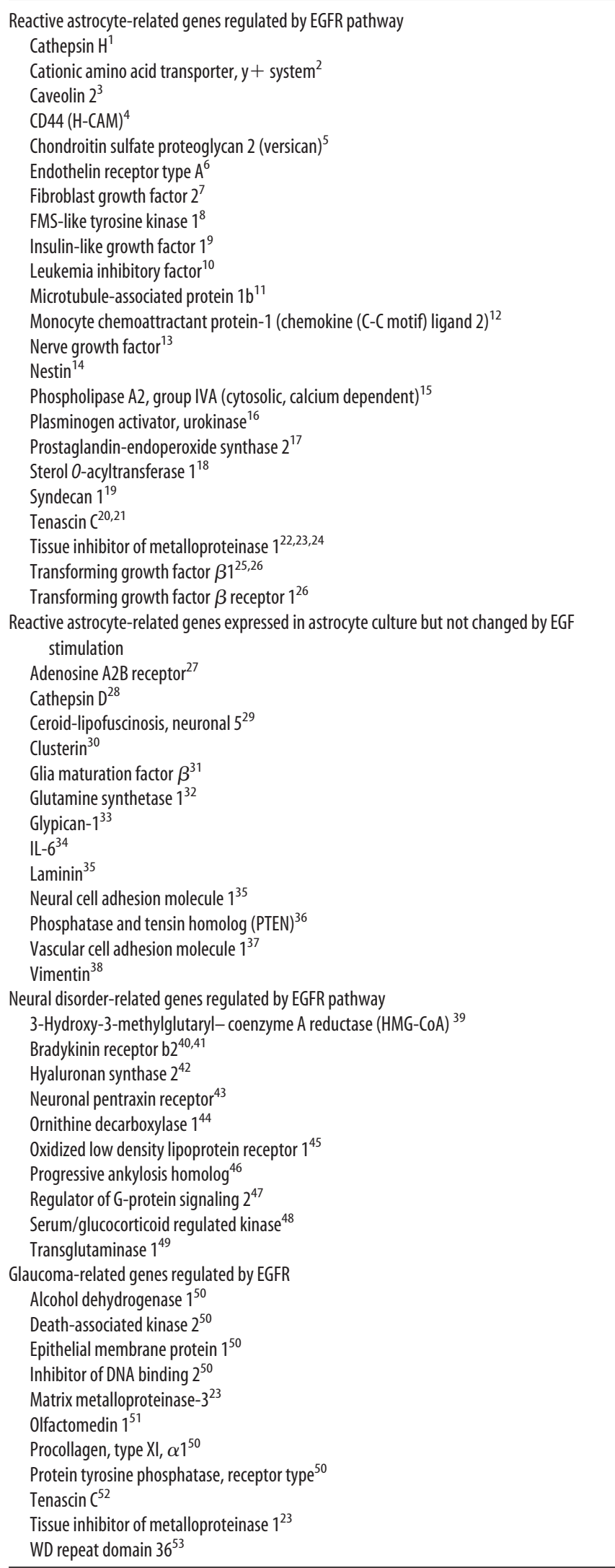

For superscripted references, see supplemental material (available at www.jneurosci.org). 
activation would be neuroprotective in an animal model of neurodegeneration.

The loss of retinal ganglion cells after optic nerve injury provides a model in which neuronal cell loss can be quantitated by retrograde labeling of the cell somas. Using a pharmacological approach to inhibit EGFR activity in astrocytes, we determined whether treatment of rats with the specific inhibitor of EGFR tyrosine kinase, AG1478 (Liu et al., 1999; Ellis et al., 2001; Liu and Neufeld, 2004a,b), can block the loss of retinal ganglion cells in an induced glaucoma model of chronic, moderately elevated IOP.

Four groups of rats with unilateral, chronic, moderately elevated IOP were studied for 7 months. Group 1 had unilaterally elevated IOP but was not treated with any drug for 7 months. Group 2 was treated with AG1478 in the drinking water throughout the 7 month period of unilaterally elevated IOP. Group 3 was not treated with any drug for the first 3.5 months of unilaterally elevated IOP and then was treated with AG1478 in the drinking water for 3.5 months. As an internal, positive control, group 4 was treated with the inhibitor of inducible nitric oxide synthase, aminoguanidine, throughout the 7 month period of unilaterally elevated IOP. Our previously reported data demonstrated that treatments with inhibitors of inducible nitric oxide synthase activity were neuroprotective in the rat glaucoma model (Neufeld et al., 1999).

Based on measurements of the amount of water intake per rat, each animal in each group drank $\sim 36 \mathrm{ml} / \mathrm{d}$. There were no differences in the amounts of water consumed on a daily basis between the untreated and AG1478-treated groups. As reported previously (Neufeld et al., 1999), aminoguanidinetreated animals drank less $(27 \mathrm{ml} / \mathrm{d})$. Based on drinking 36 $\mathrm{ml} / \mathrm{d}$ of water containing $0.1 \mathrm{~mm}$ AG1478, each animal receiving drug treatment was self-dosing at $\sim 6 \mathrm{mg} / \mathrm{kg} / \mathrm{d}$ AG1478. Figure $4 \mathrm{~A}$ demonstrates that there were no differences in body weight or weight gain between untreated and AG1478-treated animals over the 7 month dosing period. Figure $4 B$ shows the IOP in untreated and AG1478-treated rats. In the eye with normal IOP, the IOP remained low and constant over the 7 month period and was not affected by administration of AG1478. In eyes with elevated IOP, the IOP remained elevated and constant over the 7 month period and was not affected by the administration of AG1478. Administration of AG1478 did block activation of EGFR in the astrocytes of the optic nerve head (Fig. 1G,H, compare immunohistochemistry).

Figure $4 C$ compares the loss of retinal ganglion cells at 7 months in untreated and drug-treated animals. In the normal retina, the density of retinal ganglion cells in the central retina is approximately twice that of the peripheral retina. Over the 7 month period of unilaterally elevated IOP, animals that were not treated with any drug lost $\sim 20 \%$ of their retinal ganglion cells in the peripheral retina and $\sim 10 \%$ of their retinal ganglion cells in the central retina of the eyes with elevated IOP. Over the same 7 month period, animals that were treated with AG1478 essentially lost no retinal ganglion cells in the peripheral or central retinas of the eyes with elevated IOP. We interpret these pharmacological results to indicate that by blocking activation of EGFR, the reactive astrocyte phenotype was not triggered and, subsequently, there was no axonal damage and loss of retinal ganglion cells. Animals treated with aminoguanidine lost few, if any, retinal ganglion cells in the peripheral retina but $\sim 5 \%$ of their retinal ganglion cells in the central retina of the eyes with elevated IOP, consistent with results reported previously (Neufeld et al., 1999).

Figure $4 D$ demonstrates the time course of the loss of retinal ganglion cells in eyes with elevated IOP. Based on only three
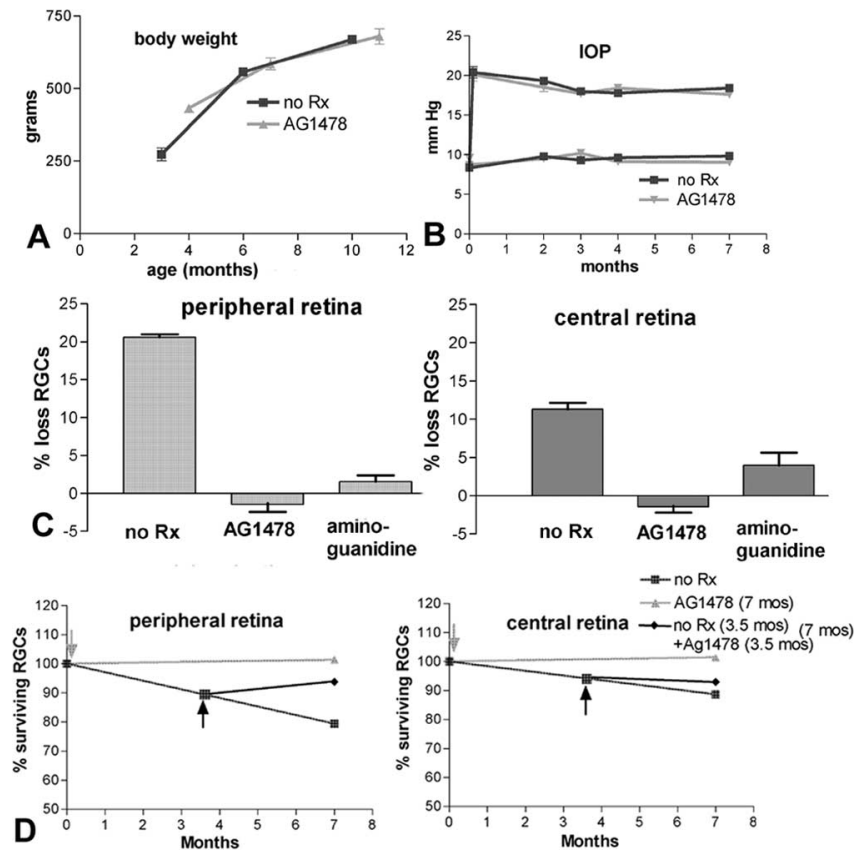

Figure 4. Administration of AG1478 prevents the loss of rat ganglion cells in a model of glaucomatous optic neuropathy. $A$, Body weight. Body weight was not different comparing untreated and AG1478-treated animals over the 7 month period. B, IOP. Low IOP was sustained in the normal eyes, and elevated IOP was sustained in the glaucomatous eyes and was not different in untreated and AG1478-treated rats. C, Loss of retinal ganglion cells at 7 months in untreated and drug-treated animals. Untreated rats lost $\sim 20 \%$ of their retinal ganglion cells in the peripheral retina and $\sim 10 \%$ of their retinal ganglion cells in the central retina of the eyes with elevated IOP. AG1478-treated rats had no loss of retinal ganglion cells in the peripheral or central retinas of the eyes with elevated IOP. Aminoguanidine-treated rats lost few retinal ganglion cells in the peripheral retina but $\sim 5 \%$ in the central retina of the eyes with elevated IOP. D, Time course of the loss of retinal ganglion cells in eyes with elevated IOP. There was an approximately linear loss of retinal ganglion cells in untreated rats but no loss in AG1478treated rats and no additional loss in peripheral and central retina of eyes with elevated IOP after starting treatment with AG1478 at 3.5 months. no Rx, Untreated.

points, the loss of retinal ganglion cells in peripheral and central retina of eyes with chronic, moderately elevated IOP was extrapolated as linear. When AG1478 was started at the time of surgical elevation of IOP, there was no loss of retinal ganglion cells in the peripheral or central retinas of eyes with elevated IOP. When retinal ganglion cell loss was allowed to proceed for 3.5 months in the absence of treatment and treatment with AG1478 was initiated and continued until 7 months, there was no additional loss of retinal ganglion cells after 3.5 months. Based on the above interpretation of the pharmacology, we believe that intervention with an inhibitor of EGFR tyrosine kinase, once retinal ganglion loss had already started, inhibited an autocrine mechanism of chronic gliosis by reactive astrocytes and no additional retinal ganglion cell loss occurred.

\section{Discussion}

Our study shows that activation of EGFR is consistently present in reactive astrocytes after different neural injuries of the optic nerve and that activation of EGFR triggers the upregulation or downregulation of a large number of genes that are associated with the reactive astrocyte phenotype. We identified a robust collection of genes that are regulated by activation of EGFR, among which there are many examples of genes associated with cellular processes that are characteristic of reactive astrocytes. Blocking EGFR function by the EGFR tyrosine kinase inhibitor prevents the loss of retinal ganglion cells in a model of glaucoma- 
tous optic neuropathy. Our results provide the first evidence identifying EGFR activation as a candidate for a novel, master, signal transduction pathway of the astrocyte activation process in response to different neural injuries and that blocking EGFR function is beneficial for the survival of neuron cells after neural injuries, at least in glaucomatous optic neuropathy.

Using an in vitro culture avoids the confounding signals associated with the immediate injury and release of cell contents, immune system activation, and heterologous cell-cell communication in vivo. Nevertheless, an in vitro model will not completely replicate the events in vivo. A few genes known to be associated with reactive astrocytes in vivo were not detected in astrocytes in vitro that were stimulated by EGFR activation. The typical astrocyte marker, GFAP, is present in vivo but was rated absent in the transcriptional profiles of astrocytes in vitro, although these astrocytes in vitro have GFAP protein (Liu and Neufeld, 2004a,b). Similar results were observed by others (Bachoo et al., 2004). We also did not find upregulation of GFAP in response to EGFR activation. The time points that we chose to examine the transcriptional profile of activated EGFR pathway genes, 4 and $12 \mathrm{~h}$, are likely too early to detect an increase in the GFAP gene expression. Also, activated EGFR produces a highly motile astrocyte in vitro but does not cause the hypertrophy of the cell soma and processes. The relationship between motility and hypertrophy of reactive astrocytes in vivo is not clear. Undoubtedly, the complete reactive astrocyte phenotype observed in vivo is a product of signals from neighboring cells such as cytokines, environmental stimuli such as reactive oxygen species, and other factors in the surrounding milieu within the injured tissue. Our findings only relate to activated EGFR pathway genes, the expressions of which may be further altered by other factors in vivo.

Depending on the in vivo insult, reactive astrocytes either reduce or exacerbate damage to neural tissue. Astrocytes support damaged neural tissues through release of neurotrophic factors, antioxidants, and degradation of extracellular deposits (WyssCoray and Mucke, 2002). In contrast, release of reactive oxygen species, cytokines, nitric oxide, and proteases by reactive astrocytes contribute to neurodegenerative disorders (Tacconi, 1998; Mrak and Griffin, 2001; Duncan and Heales, 2005). In the present study, the microarray data provide a view of the reactive astrocyte transcriptome in response to activated EGFR that suggests new experimental approaches to dissect the role of reactive astrocytes in CNS disorders.

An animal not expressing EGFR in astrocytes would be a definitive model for studying EGFR function in vivo. However, Egfr knock-outs are embryonically lethal or markedly abnormal at birth because of a critical role of EGFR in the embryologic development, involving both astrocytes and neurons (Miettinen et al., 1995; Sibilia and Wagner, 1995; Kornblum et al., 1998). We used a pharmacological approach in a glaucoma model to study the EGFR function in vivo. This model has upregulation and activation of EGFR in astrocytes and reproduces the clinical signs of glauomatous optic neuopathy with chronic loss of retinal ganglion cells (Neufeld et al., 1999). We considered using retinal ischemia or optic nerve transection as more rapid models of retinal ganglion cell loss, but these models are affected by events that are unlikely to be related to reactive astrocytes, such as the invasion of hematogenous cells and the loss of neurotrophic factors from the target neurons, respectively. The study on the glaucoma model indicates that activation of EGFR is neurodestructive, at least in the glaucomatous optic neuropathy.

Targeting EGFR activation in astrocytes may represent a potentially new therapeutic strategy against deleterious neurotoxic processes in CNS disorders. Selective inhibitors of EGFR tyrosine kinase, such as Gefitinib (IRESSA) and Erlotinib (TARCEVA), are currently used for treatment of certain forms of cancer. Perhaps these types of compounds would be useful for the treatment of neurodegenerative injuries and diseases.

\section{References}

Abraham CR (2001) Reactive astrocytes and alphal-antichymotrypsin in Alzheimer's disease. Neurobiol Aging 22:931-936.

Bachoo RM, Kim RS, Ligon KL, Maher EA, Brennan C, Billings N, Chan S, Li C, Rowitch DH, Wong WH, DePinho RA (2004) Molecular diversity of astrocytes with implications for neurological disorders. Proc Natl Acad Sci USA 101:8384-8389.

Barbeito LH, Pehar M, Cassina P, Vargas MR, Peluffo H, Viera L, Estevez AG, Beckman JS (2004) A role for astrocytes in motor neuron loss in amyotrophic lateral sclerosis. Brain Res Brain Res Rev 47:263-274.

Birecree E, Whetsell Jr WO, Stoscheck C, King Jr LE, Nanney LB (1988) Immunoreactive epidermal growth factor receptors in neuritic plaques from patients with Alzheimer's disease. J Neuropathol Exp Neurol 47:549-560.

Duncan AJ, Heales SJ (2005) Nitric oxide and neurological disorders. Mol Aspects Med 26:67-96.

Eddleston M, Mucke L (1993) Molecular profile of reactive astrocytesimplications for their role in neurologic disease. Neuroscience 54:15-36.

Ellis AG, Nice EC, Weinstock J, Levitzki A, Burgess AW, Webster LK (2001) High-performance liquid chromatographic analysis of the tyrphostin AG1478, a specific inhibitor of the epidermal growth factor receptor tyrosine kinase, in mouse plasma. J Chromatogr B Biomed Sci Appl 754:193-199.

Ferrer I, Alcantara S, Ballabriga J, Olive M, Blanco R, Rivera R, Carmona M, Berruezo M, Pitarch S, Planas AM (1996) Transforming growth factoralpha (TGF-alpha) and epidermal growth factor-receptor (EGF-R) immunoreactivity in normal and pathologic brain. Prog Neurobiol 49:99-123.

Gomez-Pinilla F, Knauer DJ, Nieto-Sampedro M (1988) Epidermal growth factor receptor immunoreactivity in rat brain. Development and cellular localization. Brain Res 438:385-390.

Hayashi Y, Yamashita J, Watanabe T (2004) Molecular genetic analysis of deep-seated glioblastomas. Cancer Genet Cytogenet 153:64-68.

Hernandez MR (2000) The optic nerve head in glaucoma: role of astrocytes in tissue remodeling. Prog Retin Eye Res 19:297-321.

Jin K, Mao XO, Sun Y, Xie L, Jin L, Nishi E, Klagsbrun M, Greenberg DA (2002) Heparin-binding epidermal growth factor-like growth factor: hypoxia-inducible expression in vitro and stimulation of neurogenesis in vitro and in vivo. J Neurosci 22:5365-5373.

Junier MP, Hill DF, Costa ME, Felder S, Ojeda SR (1993) Hypothalamic lesions that induce female precocious puberty activate glial expression of the epidermal growth factor receptor gene: differential regulation of alternatively spliced transcripts. J Neurosci 13:703-713.

Koprivica V, Cho KS, Park JB, Yiu G, Atwal J, Gore B, Kim JA, Lin E, TessierLavigne M, Chen DF, He Z (2005) EGFR activation mediates inhibition of axon regeneration by myelin and chondroitin sulfate proteoglycans. Science 310:106-110.

Kornblum HI, Hussain R, Wiesen J, Miettinen P, Zurcher SD, Chow K, Derynck R, Werb Z (1998) Abnormal astrocyte development and neuronal death in mice lacking the epidermal growth factor receptor. J Neurosci Res 53:697-717.

Li C, Hung WW (2001) Model-based analysis of oligonucleotide arrays: model validation, design issues and standard error application. Genome Biol 2:RESEARCH0032.

Li C, Wong WH (2001) Model-based analysis of oligonucleotide arrays: expression index computation and outlier detection. Proc Natl Acad Sci USA 98:31-36.

Lisovoski F, Blot S, Lacombe C, Bellier JP, Dreyfus PA, Junier MP (1997) Transforming growth factor alpha expression as a response of murine motor neurons to axonal injury and mutation-induced degeneration. J Neuropathol Exp Neurol 56:459-471.

Liu B, Neufeld AH (2000) Expression of nitric oxide synthase-2 (NOS-2) in reactive astrocytes of the human glaucomatous optic nerve head. Glia $30: 178-186$. 
Liu B, Neufeld AH (2003) Activation of epidermal growth factor receptor signals induction of nitric oxide synthase-2 in human optic nerve head astrocytes in glaucomatous optic neuropathy. Neurobiol Dis 13:109-123.

Liu B, Neufeld AH (2004a) Activation of epidermal growth factor receptor causes astrocytes to form cribriform structures. Glia 46:153-168.

Liu B, Neufeld AH (2004b) Activation of epidermal growth factor receptors directs astrocytes to organize in a network surrounding axons in the developing rat optic nerve. Dev Biol 273:297-307.

Liu W, Akhand AA, Kato M, Yokoyama I, Miyata T, Kurokawa K, Uchida K, Nakashima I (1999) 4-Hydroxynonenal triggers an epidermal growth factor receptor-linked signal pathway for growth inhibition. J Cell Sci 112:2409-2417.

Luo Y, Roth GS (2000) The roles of dopamine oxidative stress and dopamine receptor signaling in aging and age-related neurodegeneration. Antioxid Redox Signal 2:449-460.

Miettinen PJ, Berger JE, Meneses J, Phung Y, Pedersen RA, Werb Z, Derynck R (1995) Epithelial immaturity and multiorgan failure in mice lacking epidermal growth factor receptor. Nature 376:337-341.

Miller G (2005) Neuroscience. The dark side of glia. Science 308:778-781.

Monemi S, Spaeth G, DaSilva A, Popinchalk S, Ilitchev E, Liebmann J, Ritch R, Heon E, Crick RP, Child A, Sarfarazi M (2005) Identification of a novel adult-onset primary open-angle glaucoma (POAG) gene on 5 q22.1. Hum Mol Genet 14:725-733.

Mrak RE, Griffin WS (2001) Interleukin-1, neuroinflammation, and Alzheimer's disease. Neurobiol Aging 22:903-908.

Neufeld AH, Liu B (2003a) Comparison of the signal transduction pathways for the induction of gene expression of nitric oxide synthase- 2 in response to two different stimuli. Nitric Oxide 8:95-102.

Neufeld AH, Liu B (2003b) Glaucomatous optic neuropathy: when glia misbehave. Neuroscientist 9:485-495.

Neufeld AH, Sawada A, Becker B (1999) Inhibition of nitric-oxide synthase
2 by aminoguanidine provides neuroprotection of retinal ganglion cells in a rat model of chronic glaucoma. Proc Natl Acad Sci USA 96:9944-9948.

Nieto-Sampedro M, Gomez-Pinilla F, Knauer DJ, Broderick JT (1988) Epidermal growth factor receptor immunoreactivity in rat brain astrocytes. Response to injury. Neurosci Lett 91:276-282.

Norton WT (1999) Cell reactions following acute brain injury: a review. Neurochem Res 24:213-218.

Planas AM, Justicia C, Soriano MA, Ferrer I (1998) Epidermal growth factor receptor in proliferating reactive glia following transient focal ischemia in the rat brain. Glia 23:120-129.

Rezaie T, Child A, Hitchings R, Brice G, Miller L, Coca-Prados M, Heon E, Krupin T, Ritch R, Kreutzer D, Crick RP, Sarfarazi M (2002) Adultonset primary open-angle glaucoma caused by mutations in optineurin. Science 295:1077-1079.

Sibilia M, Wagner EF (1995) Strain-dependent epithelial defects in mice lacking the EGF receptor. Science 269:234-238.

Stone EM, Fingert JH, Alward WL, Nguyen TD, Polansky JR, Sunden SL, Nishimura D, Clark AF, Nystuen A, Nichols BE, Mackey DA, Ritch R, Kalenak JW, Craven ER, Sheffield VC (1997) Identification of a gene that causes primary open angle glaucoma. Science 275:668-670.

Swanson RA, Ying W, Kauppinen TM (2004) Astrocyte influences on ischemic neuronal death. Curr Mol Med 4:193-205.

Tacconi MT (1998) Neuronal death: is there a role for astrocytes? Neurochem Res 23:759-765.

Teismann P, Tieu K, Cohen O, Choi DK, Wu DC, Marks D, Vila M, JacksonLewis V, Przedborski S (2003) Pathogenic role of glial cells in Parkinson's disease. Mov Disord 18:121-129.

Wyss-Coray T, Mucke L (2002) Inflammation in neurodegenerative disease-a double-edged sword. Neuron 35:419-432.

Zhang X, Neufeld AH (2005) Activation of the epidermal growth factor receptor in optic nerve astrocytes leads to early and transient induction of cyclooxygenase-2. Invest Ophthalmol Vis Sci 46:2035-2041. 